\section{(6) OPEN ACCESS}

\title{
Surgical specimens, haemodynamics and long-term outcomes after pulmonary endarterectomy
}

\author{
Nika Skoro-Sajer, ${ }^{1}$ Gabriel Marta, ${ }^{2}$ Christian Gerges, ${ }^{1}$ Gerald Hlavin, ${ }^{3}$ Patrick Nierlich, ${ }^{2}$ \\ Shahrokh Taghavi, ${ }^{2}$ Roela Sadushi-Kolici, ${ }^{1}$ Walter Klepetko, ${ }^{2}$ Irene Marthe Lang ${ }^{1}$
}

\begin{abstract}
${ }^{1}$ Department of Internal Medicine II, Division of Cardiology, Vienna General Hospital, Medical University of Vienna, Vienna, Austria ${ }^{2}$ Department of Cardiothoracic Surgery, Vienna General Hospital, Medical University of Vienna, Vienna, Austria ${ }^{3}$ Section of Medical Statistics, Vienna General Hospital, Medical University of Vienna Vienna, Austria
\end{abstract}

\section{Correspondence to} Professor Irene Marthe Lang, Department of Internal Medicine II, Division of Cardiology, Medical University of Vienna, Waehringer Guertel 18-20, Vienna 1090, Austria; irene.lang@meduniwien.ac.at

Received 18 April 2013 Revised 8 August 2013 Accepted 22 August 2013 Published Online First 19 September 2013

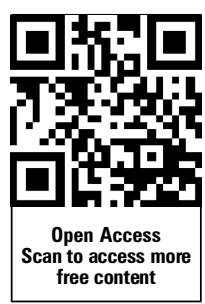

\section{SLinked}

http://dx.doi.org/10.1136/ thoraxjnl-2013-203413

- http://dx.doi.org/10.1136/

thoraxjnl-2013-204193

To cite: Skoro-Sajer $\mathrm{N}$ Marta G, Gerges C, et al

Thorax 2014;69:116-122.

\section{ABSTRACT}

Background Chronic thromboembolic pulmonary hypertension is surgically curable by pulmonary endarterectomy (PEA). It is unclear whether PEA impacts primarily steady state right ventricular afterload (ie, pulmonary vascular resistance (PVR)) or pulsatile right ventricular afterload (ie, pulmonary arterial compliance $\left.\left(C_{P A}\right)\right)$. Our objectives were to (1) quantify PEA specimens and measure the impact of PEA on PVR and $C_{P A}$ in a structure/function study and (2) analyse the effects of haemodynamic changes on long-term survival/ freedom of lung transplantation in an outcome study. Methods Thrombi were laid out, weighed, photographed and measured. PVR, $C_{P A}$ and resistance times compliance (RC-time) were assessed at baseline, within 4 days after PEA ('immediately postoperative') and 1 year after PEA, in 110 consecutive patients who were followed for $34.5(11.9 ; 78.3)$ months.

Results Lengths and numbers of PEA specimen tails were inversely correlated with immediate postoperative PVR $(p<0.0001, r=-0.566 ; p<0.0001, r=-0.580)$. PVR and $C_{P A}$ normalised immediately postoperatively while RC-time remained unchanged. Immediate postoperative PVR was the only predictor of long-term survival/freedom of lung transplantation $(p<0.0001)$. Patients with immediate postoperative PVR $<590$ dynes.s. $\mathrm{cm}^{-5}$ had better long-term outcomes than patients with PVR $\geq 590$ dynes.s. $\mathrm{cm}^{-5}(p<0.0001$, respectively). Conclusions PEA immediately decreased PVR and increased $C_{P A}$ under a constant RC-time. However, immediate postoperative PVR was the only predictor of long-term survival/freedom of lung transplantation. Our study confirms the importance of a complete, bilateral surgical endarterectomy. Low PVR measured immediately postoperative predicts excellent long-term outcome.

\section{INTRODUCTION}

Pulmonary endarterectomy (PEA) is the treatment of choice for chronic thromboembolic pulmonary hypertension $(\mathrm{CTEPH})^{1}$ with a periprocedural mortality rate of $<5 \%$ in Europe today, ${ }^{2}$ nearly normalised haemodynamics and substantial improvement in clinical symptoms in the majority of patients. ${ }^{1-4}$ Outcomes after PEA have been estimated on the basis of postoperative pulmonary vascular resistance (PVR) measurements, with a PVR of 500 dynes.s.cm ${ }^{-5}$ as a threshold of favourable outcome in several databases. ${ }^{12}$ Right ventricular (RV) afterload bears a steady component (represented by PVR) and an oscillatory component (the opposition that the ventricle encounters to

\section{Key messages}

What is the key question?

- What are the effects of pulmonary endarterectomy (PEA) on pulmonary vascular resistance and compliance in chronic thromboembolic pulmonary hypertension (CTEPH), and what is the prognostic impact of these effects?

\section{What is the bottom line?}

- Postoperative pulmonary vascular resistance assessed in the intensive care unit is a strong predictor of long-term survival/freedom of lung transplantation in patients with CTEPH undergoing PEA.

\section{Why read on?}

- This is the first prospective longitudinal study confirming a correlation among characteristics of the surgical specimen, haemodynamics and long-term right ventricular recovery in patients with CTEPH undergoing PEA.

maintain forward flow and pulsatile components of flow). Pulmonary arterial compliance $\left(\mathrm{C}_{\mathrm{PA}}\right)$ relates to oscillatory load with potentially greater prognostic importance than resistance. ${ }^{5} 6 \mathrm{RV}$ hydraulic load is determined by the dynamic interaction between PVR and $\mathrm{C}_{\mathrm{PA}}{ }^{7}$. The product of resistance (PVR) and $\mathrm{C}_{\mathrm{PA}}$ is consistently inversely related and remains unaltered at approximately $0.7 \mathrm{~s}$ in various types of pulmonary hypertension $(\mathrm{PH}) .{ }^{8}$ We have recently reported that patients with persistent exertional dyspnoea after successful PEA display an abnormal pulmonary haemodynamic response to exercise, characterised by increased PVR and decreased $\mathrm{C}_{\mathrm{PA}}{ }^{7}$ Because PEA mainly affects major vessels that are surgically accessible, and $C_{P A}$ appears to be determined by the larger vessel compartment, ${ }^{9}$ we hypothesised that PEA primarily increases $\mathrm{C}_{\mathrm{PA}}$, which may then also be an important predictor of prognosis in CTEPH. Therefore, we quantified thrombus, measured the impact of PEA on haemodynamics in a 'structure/function' study and analysed which RV afterload parameter (PVR, $\mathrm{C}_{\mathrm{PA}}$ or resistance times compliance (RC-time)) is the best predictor of long-term survival/freedom of lung transplantation in an outcome study. 


\section{METHODS}

\section{Patients and clinical assessments}

The study was carried out at the Pulmonary Hypertension Unit, Vienna General Hospital, a national referral centre for CTEPH in Austria. Diagnosis of CTEPH, criteria for PEA and surgical procedures were based on previously published standards. ${ }^{10} 11$ Patients undergoing bilateral PEA at our institution were included in the study and were followed for $34.5(11.9 ; 78.3)$ months.

Right heart catheterisation (RHC) was obtained on three occasions: at baseline, within 4 days after PEA in the intensive care unit prior to removal of the intraoperative Swan-Ganz and left atrial catheters ('immediate postoperative') when patients were off vasopressors, and 1 year after PEA ('1-year follow-up (FU)'). During baseline catheterisation, left ventricular enddiastolic pressures were measured to validate wedge pressures. During PEA, a left atrial catheter was inserted intrapericardially via the right upper lobe vein in the left atrium, and out through the skin under the right costal arch. The catheter allowed post-PEA measurements of left atrial pressures. The catheter was pulled within a few hours and a maximum of 4 days, after a final haemodynamic measurement. After the 1-year FU, patients were seen on a yearly basis as outpatients. Additional RHCs were indicated on the basis of clinical signs and symptoms.

\section{Haemodynamic definitions}

PVR was calculated by mean pulmonary arterial pressure (mPAP)—-mean pulmonary capillary wedge pressure/cardiac output (CO) multiplied by 80 for dynes.s. $\mathrm{cm}^{-5}$.

$\mathrm{C}_{\mathrm{PA}}$ was calculated as stroke volume $(\mathrm{CO} /$ heart rate) divided by systolic PAP-diastolic PAP. The RC-time (product of resistance $(\mathrm{R}=\mathrm{PVR})$ and compliance $\left(\mathrm{C}_{\mathrm{PA}}\right)$ ) was calculated as previously described ${ }^{8}$ and expressed in seconds.

Persistent/recurrent $\mathrm{PH}$ was defined as $\mathrm{mPAP} \geq 25 \mathrm{mmHg}$ and PVR $\geq 400$ dynes.s.cm ${ }^{-5}$ at the routine 1 -year FU RHC. ${ }^{11}$

\section{Analysis of PEA specimens}

The Jamieson classification system ${ }^{3}$ was used to describe the four major types of CTEPH. Jamieson type I is semiorganised or organised thrombus that begins in the main or lobar arteries; type II is organised thrombus and intimal thickening proximal to segmental arteries; type III is intimal thickening and fibrosis in the distal segmental arteries; and type IV is distal arteriolar vasculopathy with no intraluminal disease. If there was a discrepancy in CTEPH type between left and right specimen, the most proximal thrombus determined the final classification.

Starting 2005, semiquantitative assessment of consecutive PEA specimens, labelled as 'thrombi', was performed. Thrombi were laid out, weighed and photographed $(n=69)$. Organised small-vessel thrombus ('thrombus tails') was defined as white dissectate, $<2 \mathrm{~mm}$ in thickness and at least $2 \mathrm{~mm}$ in length. Thrombus tails were counted and measured in centimetres. Those analysing PEA specimens (GM and CG) were blinded to haemodynamic data.

\section{Statistical analysis}

Continuous parameters were summarised by means \pm SDs, or in the case of skewed distributions, by medians (25\% percentile; $75 \%$ percentile). Discrete data were presented as counts. Deltas $(\Delta s)$ were calculated as the difference between values at the immediate postoperative RHC and baseline RHC, and were depicted as vectors for each patient.
Disease-specific survival was defined as freedom from lung transplantation or death from right heart failure, which was defined as death in the intensive care unit under the signs of RV pump failure, or was based on an autopsy report and written confirmation by the primary care physician if the patient died outside of the hospital. For comparisons, $t$ tests were performed using the Bonferroni correction. The impact of haemodynamic parameters and thrombus characteristics on survival and $\mathrm{PH}$ recurrence was analysed by Cox proportional hazard models and logistic regression models. Models were adjusted for age, sex and baseline PVR. All tests regarding the hazard rate were subjected to Bonferroni correction. Time dependent receiver operator characteristic curves (ROC) at 1, 3 and 5 years were plotted for all risk factors, which were significant $(\alpha=0.05)$ in the Cox models, using the R package 'pROC'12 to determine area under the curve, specificity and sensitivity of the optimal cut-offs (according to Youden's index). Regarding the outcome variable status, Kaplan-Meier-survival curves were plotted.

In addition, Fisher's exact tests were calculated for baseline PVR $>1000$ dynes.s. $\mathrm{cm}^{-5}$ as predictor of survival. Spearman correlation coefficients among change of $\mathrm{C}_{\mathrm{PA}}$, immediate postoperative PVR and thrombus size (ie, the total number of thrombus tails and their total length per patient) were calculated. p Values below 0.05 were considered statistically significant. The statistical computing environment R V.2.13.1 was used. $^{13}$

The Ethics Committee of the Medical University of Vienna approved the study, and participating patients signed informed consents.

\section{RESULTS}

\section{Baseline characteristics}

A total of 110 patients undergoing PEA between May 1994 and December 2010 were included in the study. Patients' baseline characteristics are summarised in table 1 .

All patients were on standard medical therapy consisting of oral anticoagulation (international normalised ratio 2.0-3.0), digitalis, diuretics and other supportive treatments including birth control and vaccinations. Five patients who were referred from other centres received preoperative pulmonary arterial hypertension (PAH)-specific therapies (sildenafil, bosentan, inhaled iloprost) that were terminated at the time of PEA. Patients underwent surgery $129 \pm 362$ days after diagnosis. CTEPH types ${ }^{3}$ are listed in table 1.

Table 1 Baseline patient characteristics

\begin{tabular}{ll}
\hline & $\mathbf{n}$ \\
\hline Age (years) & $\mathbf{1 1 0}(\mathbf{1 0 0 \% )}$ \\
Gender (f/m) & $56 \pm 14$ \\
BMI & $56 / 54$ \\
WHO functional class (II/III/IV) & $26 \pm 4$ \\
6-MWD (m) & $5 / 76 / 29$ \\
Time from first symptoms to diagnosis (months) & $390 \pm 119$ \\
Time to PEA (days) & $26 \pm 36$ \\
Medical treatment prior to PEA (n=number of patients) & $129 \pm 362$ \\
CTEPH types I/II/III/IV & $5(0.05)$ \\
\hline The data are presented as means \pm SD. Discrete data are presented as counts. \\
6-MWD, 6-min walking distance; BMl, body mass index; CTEPH, chronic \\
thromboembolic pulmonary hypertension; PEA, pulmonary endarterectomy.
\end{tabular}


A

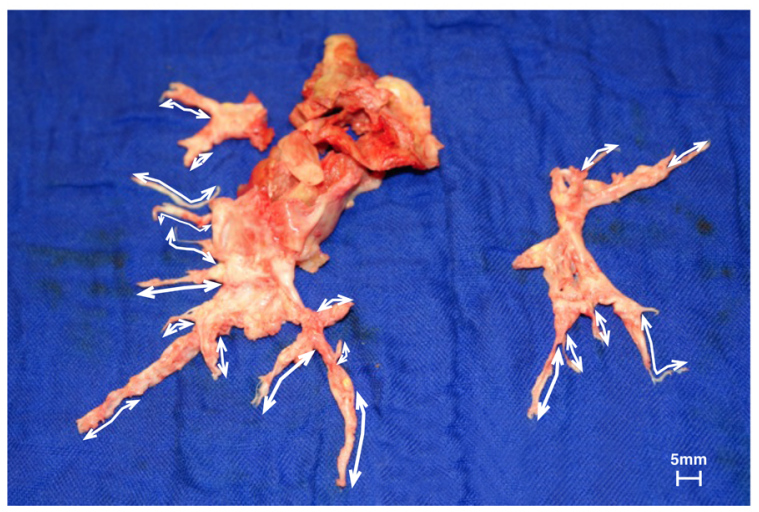

B

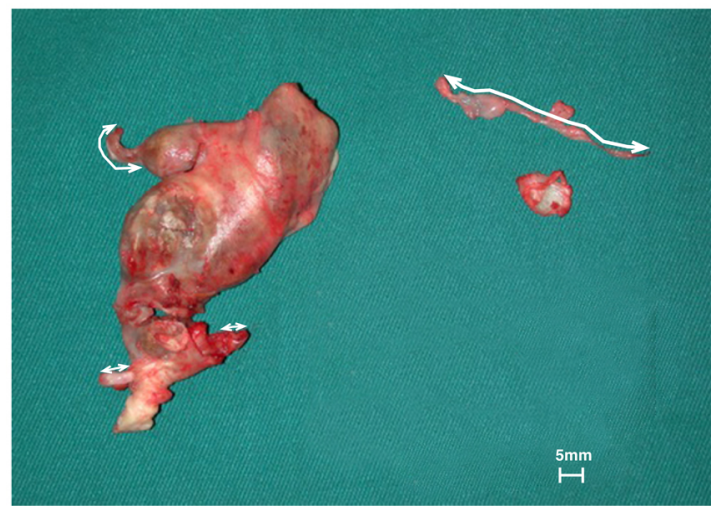

Figure 1 Illustration of how numbers and lengths of tails were accounted for. Tails were defined as dissectates not exceeding 2 mm in thickness and at least $2 \mathrm{~mm}$ in length (measured tails in this specimen are pointed out by white double-headed arrows). For the analysis, thrombus tails were counted and their total length was expressed in centimetres. The following two examples represent a 'good' (A) and a 'poor' (B) surgical specimen. (A) Surgical specimen of patient \#101 with an immediate postoperative pulmonary vascular resistance (PVR) of $190 \mathrm{dynes.s.cm}^{-5}$ and 19 thrombus tails (total tail length $24.5 \mathrm{~cm}$ ). (B) Surgical specimen of patient \#14 with an immediate postoperative PVR of $640 \mathrm{dynes}^{\mathrm{s} . \mathrm{cm}^{-5}}$ and only four thrombus tails (total tail length $8.1 \mathrm{~cm}$ ).

\section{Analysis of PEA specimens}

In all, $11 \pm 3$ thrombus tails were counted per surgical specimen, measuring $19 \pm 5 \mathrm{~cm}$ in total length. Figure 1 shows two representative examples of surgical specimen analyses. Total numbers and lengths of thrombus tails were inversely correlated with immediate postoperative PVR $(\mathrm{r}=-0.58, \mathrm{p}<0.0001 ; \mathrm{r}=-0.57$, $\mathrm{p}<0.0001$; figure $2 \mathrm{~A}, \mathrm{~B}$ ) and $\Delta \mathrm{C}_{\mathrm{PA}}$ (from baseline to immediately postoperative, $\mathrm{r}=0.44, \mathrm{p}=0.002$; and to 1 year $\mathrm{FU}$, $\mathrm{r}=0.44, \mathrm{p}=0.002$ ). Thrombus weights varied widely and did not correlate with haemodynamic parameters (data not shown).

\section{Haemodynamic changes as a consequence of PEA}

Haemodynamic assessment within 4 days after PEA (2 \pm 2 days) was performed in 104 patients, with left atrial pressures serving to assess postoperative PVRs. The remaining six patients are \#19, \#22, \#42, \#43 (operated in 1996), \#72 (operated in 2004) and \#81. Immediate postoperative data of these patients were not available because left atrial catheters were pulled before a final haemodynamic evaluation.

A

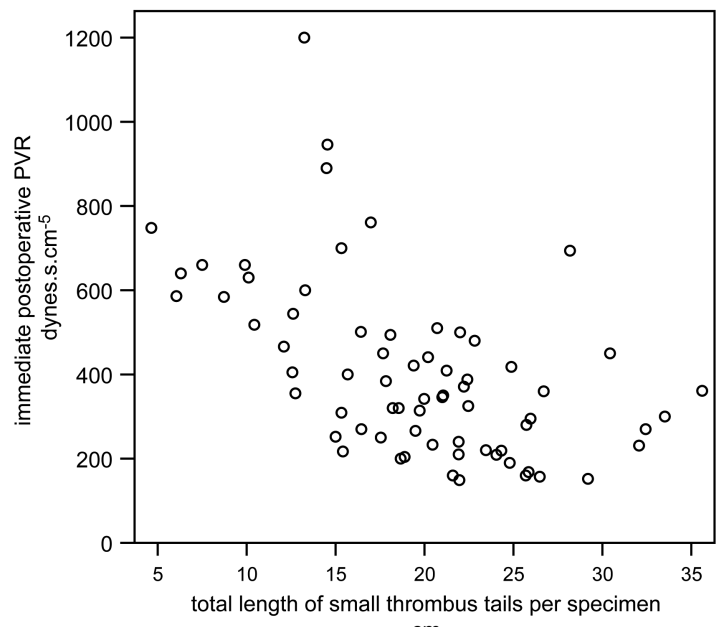

Haemodynamic parameters at baseline, immediate postoperative and at 1-year FU are listed in table 2.

PVR, $C_{P A}, R C$-time and stroke volume

PVR decreased from preoperative $770.4(583 ; 1011)$ to 368 $(251 ; 516)$ dynes.s.cm ${ }^{-5}$ immediate postoperative $(p<0.001$, figure 3A). The change of PVR between immediate postoperative and 1-year FU was not significant $(p=0.22) . C_{P A}$ changed significantly from baseline to immediate postoperative, without further changes to 1-year FU (figure 3B). On average, RC-time decreased after PEA, albeit not significantly: RC-time $=0.72 \pm 0.71 \mathrm{~s}$ (baseline), RC-time $=0.60 \pm 0.3 \mathrm{~s}$ (immediate postoperative) and $\mathrm{RC}$-time $=0.59 \pm 0.34 \mathrm{~s}$ (1-year FU; figure $3 \mathrm{C}$; paired t test: $\mathrm{p}=0.13$ from baseline to immediate postoperative, and $p=0.32$ from baseline to 1-year FU). The change of RC-time was not different between patients with persistent/recurrent $\mathrm{PH}$ compared with patients with no $\mathrm{PH}$ (immediately postoperative $p=0.81$, at 1 year $p=0.64$ ).

\section{B}

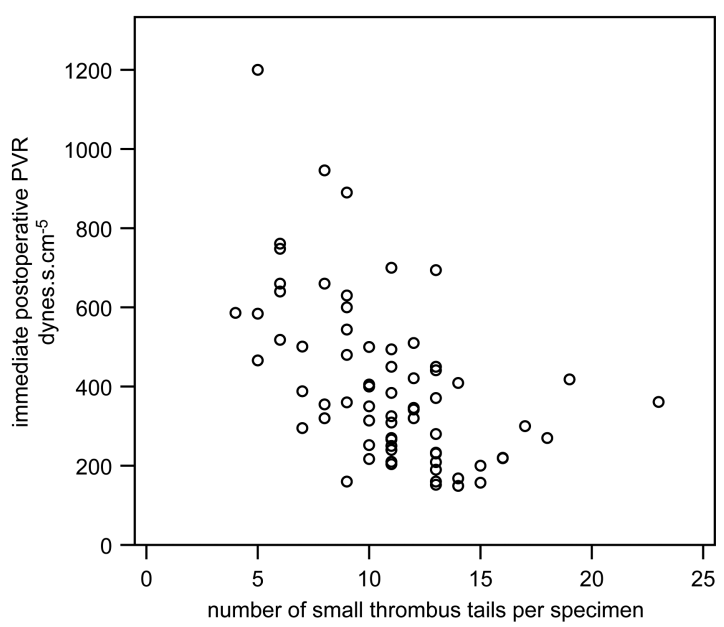

Figure 2 Scatter plots of immediate postoperative pulmonary vascular resistance (PVR) against the cumulative length of surgically extracted thrombus tails in centimetres $(p<0.0001, r=-0.566(A))$ and against the cumulative number of surgically extracted thrombus tails $(p<0.0001$, $r=-0.580(B))$. 
Table 2 Haemodynamic parameters assessed at baseline, immediate postoperative and at 1-year follow-up

\begin{tabular}{llll}
\hline & $\begin{array}{l}\text { Baseline } \\
\mathbf{n}=110\end{array}$ & $\begin{array}{l}\text { Immediate } \\
\text { postoperative } \\
\mathbf{n}=104\end{array}$ & $\begin{array}{l}\text { 1-year FU } \\
\mathbf{n}=71\end{array}$ \\
\hline PVR (dynes.s.cm $\left.{ }^{-5}\right)$ & $770(583 ; 1011)$ & $368(251 ; 516)^{* *}$ & $280(186 ; 472)^{* *}$ \\
$C_{P A}(\mathrm{~mL} / \mathrm{mm} \mathrm{Hg})$ & $1.0(0.8 ; 1.4)$ & $2.2(1.5 ; 3.2)^{* *}$ & $2.7(1.4 ; 3.8)^{* *}$ \\
RC-time(s) & $0.72 \pm 0.71$ & $0.60 \pm 0.3$ & $0.59 \pm 0.34$ \\
SV (mL) & $58.4 \pm 16.8$ & $61.1 \pm 20.9$ & $71.6 \pm 18.6 * *$ \\
\hline
\end{tabular}

PVR and $C_{P A}$ are presented as medians ( $25 \%$ percentile; $75 \%$ percentile), RC-time and $\mathrm{SV}$ as means \pm SDs.

${ }^{* *} p<0.001$

$\mathrm{C}_{\mathrm{PA}}$, pulmonary arterial compliance; FU, follow-up; PVR, pulmonary vascular

resistance; RC-time, resistance times compliance; SV, stroke volume.

Stroke volume increased from baseline to 1-year FU, while it did not change significantly from baseline to immediate postoperative (figure 3D).
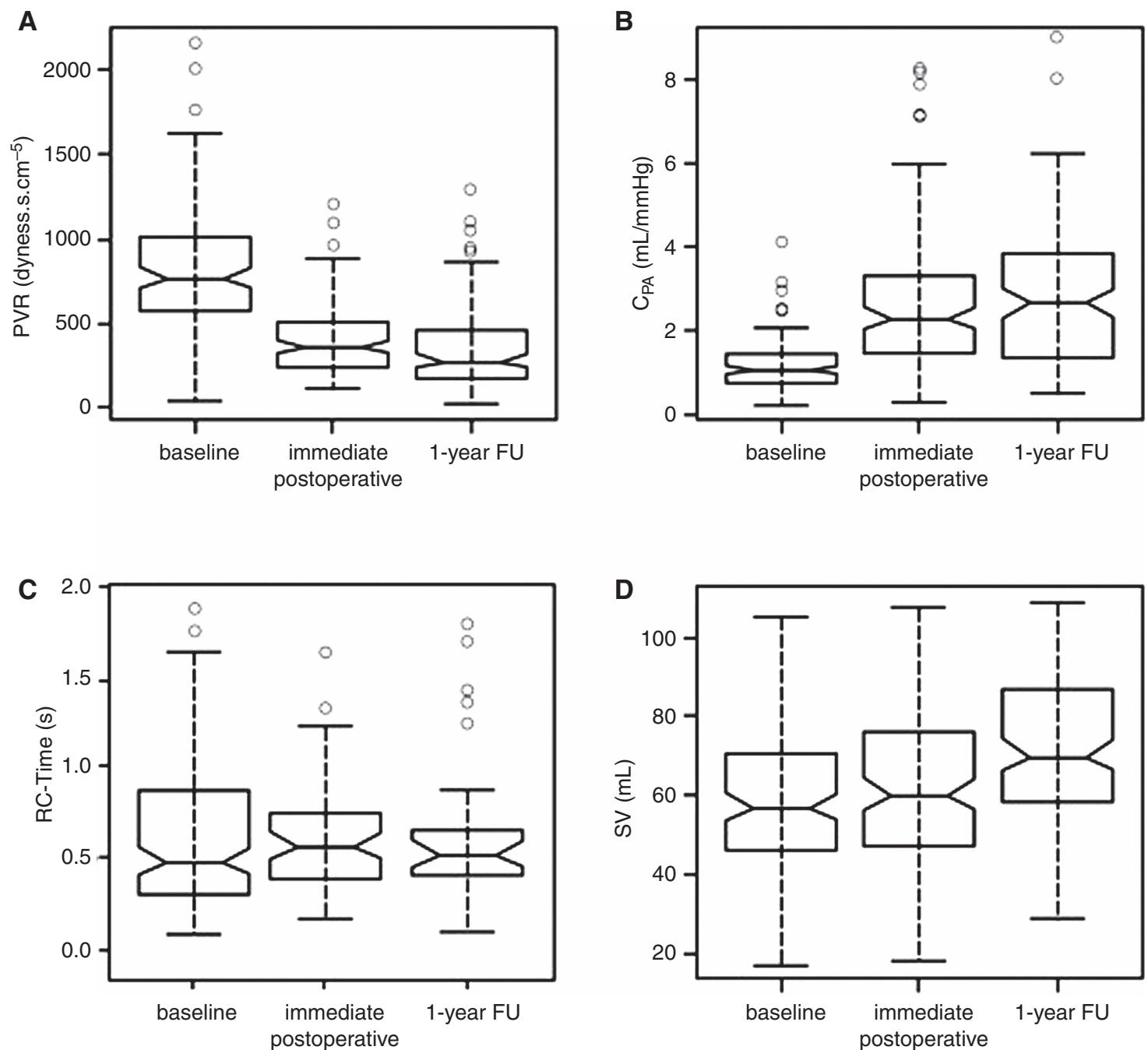

Figure 3 Box plots of steady and pulsatile flow parameters at baseline, immediately postoperative and at 1-year follow-up (FU). (A) Pulmonary vascular resistance (PVR) decreased from preoperative $770.4(583.2 ; 1011)$ to $368.5(250.5 ; 516)$ immediately postoperative, and to $280(186.3$; 472 ) dynes.s. $\mathrm{cm}^{-5}$ at 1 -year FU $\left(p<0.001, p<0.001\right.$, ie, respective changes from baseline). (B) Pulmonary arterial compliance $\left(C_{P A}\right)$ changed from 1.0 $(0.8 ; 1.4)$ to $2.1(1.5 ; 3.2)$ immediately postoperative, and to $2.7(1.4 ; 3.8) \mathrm{mL} / \mathrm{mm} \mathrm{Hg}$ at 1 -year FU $(p<0.001$ and $p<0.001)$. (C) Resistance times compliance (RC-time) did not change significantly during the observation period: RC-time $=0.72+0.71 \mathrm{~s}$ (baseline), RC-time $=0.60+0.3 \mathrm{~s}$ (immediately postoperatively) and RC-time $=0.59+0.34 \mathrm{~s}$ (1-year FU; $p=0.13$ and $p=0.32$ ). (D) Stroke volume (SV) increased from preoperative $58.4 \pm 16.8$ to 61.1 \pm 20.9 immediately postoperative and to $71.6 \pm 18.6 \mathrm{~mL}$ at 1 -year $\mathrm{FU}(\mathrm{p}=\mathrm{NS}$ and $\mathrm{p}<0.001)$. catheterisations

Figure 4 depicts vectors from the origin that indicate the change $(\Delta)$ in both PVR and $\mathrm{C}_{\mathrm{PA}}$ between catheterisations at baseline and immediate postoperative to discriminate poor 'PVR-responders' from poor ' $\mathrm{C}_{\mathrm{PA}}$-responders'. The majority of vectors $(n=78,75 \%)$ point to the top-left quadrant indicating that if PVR decreases, $\mathrm{C}_{\mathrm{PA}}$ increases concordantly. The five vectors ending in the top-right quadrant ('PVR non-responders') represent five patients with persistent/recurrent $\mathrm{PH}$, with one patient dying from right heart failure. In the left-bottom quadrant (concordant decreases of PVR and $\mathrm{C}_{\mathrm{PA}}$ ) are 21 vectors representing predominantly female patients, of whom 14 patients (67\%) had persistent/ recurrent $\mathrm{PH}$, with seven deaths (33\%). In this group were three patients with ventriculo-atrial (VA) shunts (out of a total of four patients with (VA) shunts among the 110 patients).

\section{Survival}

Overall survival showed 1-, 3 - and 5-year cumulative rates of $92 \%, 89 \%, 85 \%$ and $61 \%$ at 10 years.

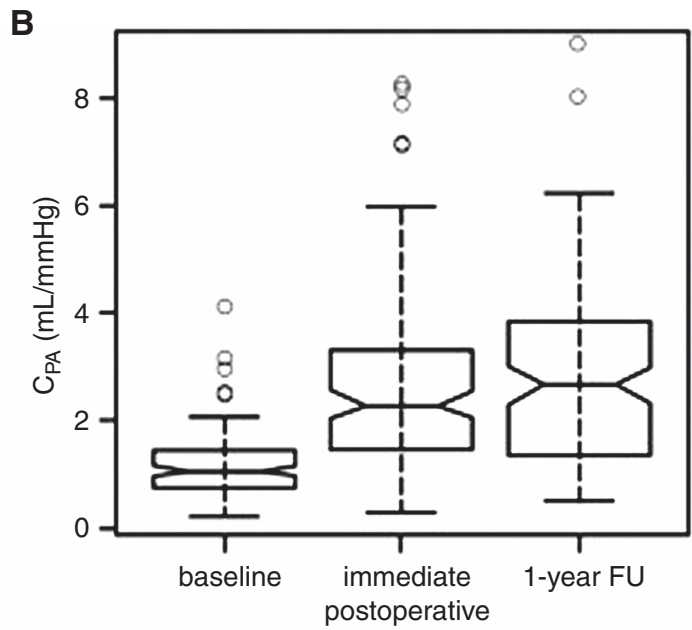

Vector diagram indicating the change in both $\mathrm{R}$ and $\mathrm{C}$ between 
Figure $4 \Delta$ Pulmonary vascular resistance (PVR) and $\Delta$ pulmonary arterial compliance $\left(\mathrm{C}_{\mathrm{PA}}\right)$ represented as vectors from the origin, indicating changes in both PVR and $C_{P A}$ between catheterisations at baseline and immediately postoperative. All patients' vectors with persistent/ recurrent pulmonary hypertension $(\mathrm{PH})$ in the left-upper quadrant are localised within the circled area (representing in $C_{P A}$ and PVR). cases with only a minor improvement

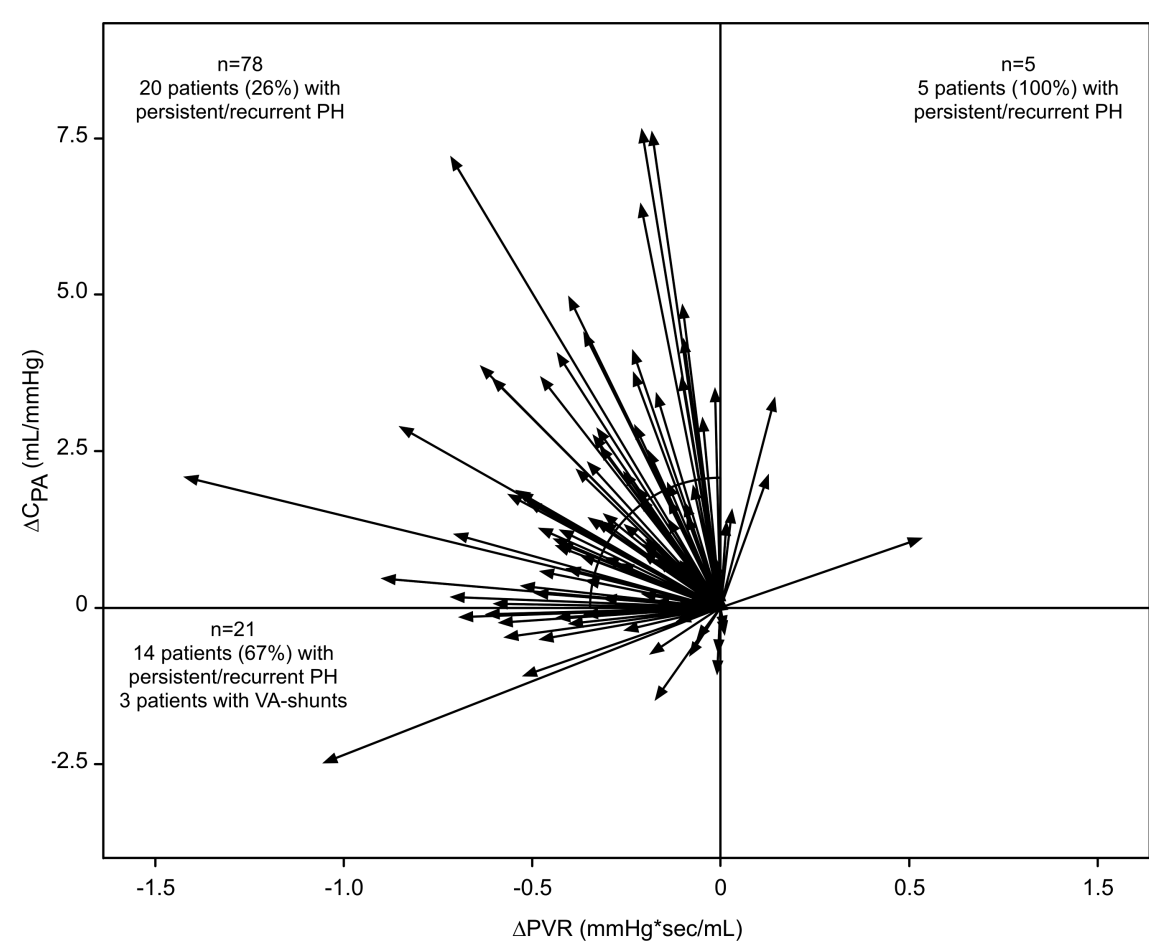

Perioperative inhospital death occurred in five patients (three female patients, 5\%) at $11 \pm 14$ days after PEA (table 3 ) due to right heart failure. Deceased patients had higher baseline PVRs $\left(1090 \pm 502\right.$ dynes.s. $\left.\mathrm{cm}^{-5}\right)$ and higher immediate postoperative PVRs $\left(821 \pm 501\right.$ dynes.s.cm $\left.{ }^{-5}\right)$. Three further patients died within the first year ( $163 \pm 117$ days postoperatively). Mean immediate postoperative PVR was $587 \pm 147$ dynes.s.cm ${ }^{-5}$ in these cases.

\section{Predictors of outcome}

During the observation 21 events occurred. Two patients died from malignancies and were excluded from the analyses of disease-specific survival.

Baseline PVR $>1000$ dynes.s. $\mathrm{cm}^{-5}$ was a weak predictor of 1-, and 3- year survival (Fisher's exact test $\mathrm{p}=0.05, \mathrm{p}=0.06$ ).

In all, 28 patients $(26 \%)$ had a PVR $>1000$ dynes.s.cm ${ }^{-5}$ and a high risk for postoperative mortality. ${ }^{1}$ Of these, eight patients (29\%) died within the observation period, with immediate postoperative PVRs of $765 \pm 237$ dynes.s. $\mathrm{cm}^{-5}$.

Cox regression analysis revealed immediate postoperative PVR as the strongest independent predictor of long-term survival/freedom of lung transplantation (HR 1.005; 95\%, HR confidence limits 1.003 to $1.006 ; \mathrm{p}<0.0001)$. Immediate

Table 3 Haemodynamic variables of patients with early mortality (within 31 days after PEA), at baseline and immediately postoperative

\begin{tabular}{lll}
\hline & Baseline & Immediately postoperative \\
\hline PVR (dynes.s.cm $\left.{ }^{-5}\right)$ & $1090 \pm 502$ & $821 \pm 254$ \\
$C_{P A}(\mathrm{~mL} / \mathrm{mm} \mathrm{Hg})$ & $0.92 \pm 0.3$ & $1.5 \pm 1.5$ \\
$\mathrm{SV}(\mathrm{mL})$ & $44.6 \pm 16.7$ & $40.6 \pm 27$ \\
\hline
\end{tabular}

The data are presented as means \pm SDs.

$\mathrm{C}_{\mathrm{PA}}$, pulmonary arterial compliance; $\mathrm{PEA}$, pulmonary endarterectomy; $\mathrm{PVR}$, pulmonary vascular resistance; $S V$, stroke volume. postoperative PVR $\geq 590$ dynes.s.cm ${ }^{-5}$ can predict death/lung transplantation in patients after PEA (figure 5).

Patients with immediate postoperative PVR $<590$ dynes.s. $\mathrm{cm}^{-5}$ had a better long-term outcome than patients with PVR $\geq 590$ dynes.s.cm ${ }^{-5} \quad(p<0.0001$; figure 6A). The absolute value of immediately postoperative PVR predicted survival. Patients with immediate postoperative PVR $<290$ dynes.s. $\mathrm{cm}^{-5}$ had a better survival than patients with PVR between 292 and 450 dynes.s. $\mathrm{cm}^{-5}$, and those with immediate postoperative PVR $>450$ dynes.s. $\mathrm{cm}^{-5}$ (figure 6B). None of the patients with

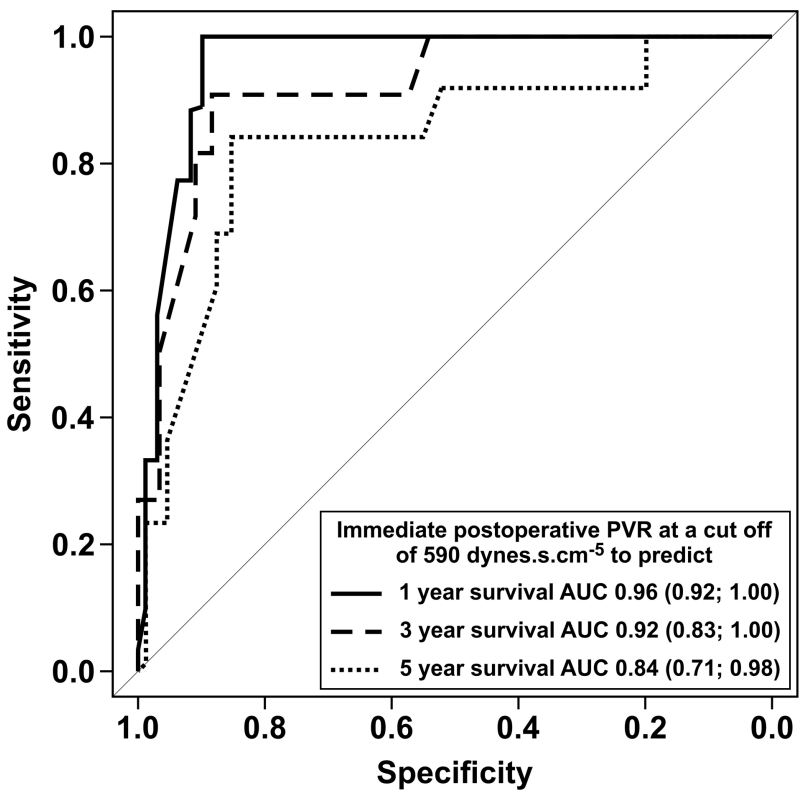

Figure 5 Receiver operator characteristics curve identifying an immediate postoperative pulmonary vascular resistance (PVR) of 590 dynes.S. $\mathrm{cm}^{-5}$ as the PVR threshold with greatest areas under the curve for likelihood of survival at 1, 3 and 5-year follow-ups. 
A

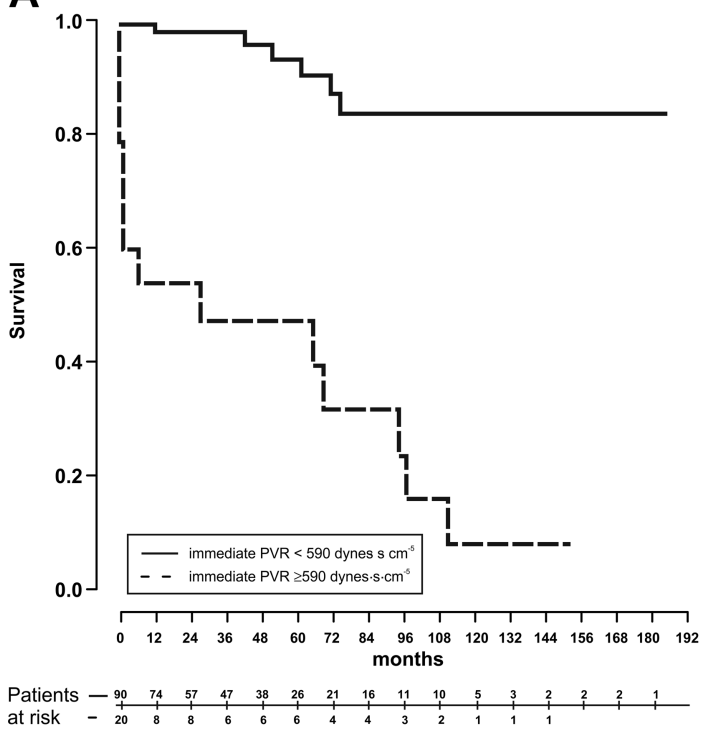

B

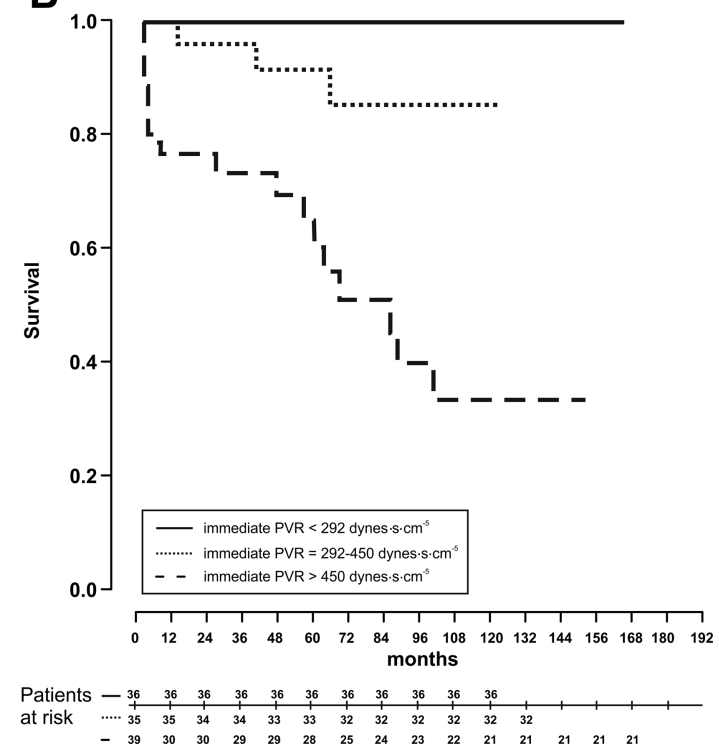

Figure 6 (A) Kaplan-Meier survival curves in patients with operated chronic thromboembolic pulmonary hypertension (CTEPH) and immediate postoperative pulmonary vascular resistance (PVR) $\geq 590$ dynes.s. $\mathrm{cm}^{-5}$ compared with patients with immediate postoperative PVR $<590$ dynes.s. $\mathrm{cm}^{-5}$. Patients with immediate postoperative PVR $<590$ dynes.s. $\mathrm{cm}^{-5}$ had a better long-term outcome than patients with $\mathrm{PVR} \geq 590$ dynes.s.cm ${ }^{-5}$ $(p<0.0001)$. (B) Kaplan-Meier survival curves in patients with operated CTEPH by tertiles of immediate PVR $(p<0.001$, respectively).

an immediate postoperative PVR $<290$ dynes.s. $\mathrm{cm}^{-5}$ died or underwent lung transplantation within the observation period.

The numbers and lengths of thrombus tails were predictors of survival/freedom of lung transplantation ( $\mathrm{HR} 0.81, \mathrm{p}=0.02$; HR $0.9 ; \mathrm{p}=0.01$, respectively).

Neither $C_{P A}$ nor RC-time showed a significant influence on survival.

\section{Predictors of persistent/recurrent $\mathrm{PH}$}

No baseline haemodynamic parameter, but only immediate postoperative PVR (HR 1.004; 95\%, HR confidence limits: 1.0032 to $1.0056 ; \mathrm{p}<0.0001)$ was an independent predictor of persistent/recurrent $\mathrm{PH}$.

The numbers and lengths of thrombus tails were predictors of persistent/recurrent PH $(n=25$; Log OR 0.74, $p=0.0026$; Log OR $0.86, p=0.002$, respectively).

\section{DISCUSSION}

Our data show that (i) PEA immediately decreased PVR and increased $\mathrm{C}_{\mathrm{PA}}$, under a constant RC-time. However, (ii) immediate postoperative PVR was the only predictor of long-term sur$\mathrm{vival} /$ freedom of lung transplantation and (iii) patients from whom more and longer thrombus tails were removed had better postoperative haemodynamics and were less likely to develop persistent/recurrent PH. The outcome of operable CTEPH is best predicted immediately after PEA.

PVR and $\mathrm{C}_{\mathrm{PA}}$ in patients with CTEPH are determined by major-vessel thromboembolic obstruction, small-vessel arteriopathy, right-heart function and $\mathrm{CO} .{ }^{14}$ Our structure/function study illustrated that the number of small thrombus tails determines normalisation of PVR after successful PEA, thus impacting short-term and long-term mortality. ${ }^{1}$ Specimen assessment takes into account both the technical success of PEA and anatomical CTEPH type that has previously been correlated with outcomes. ${ }^{15}$ In contrast to the systemic arterial system where compliance is mainly located in the proximal aorta, compliance of the pulmonary arterial tree in CTEPH is less well studied. ${ }^{16-18} \mathrm{C}_{\mathrm{PA}}$ reflects pulsatile load of the right ventricle and appears to be mainly determined by large vessels. ${ }^{9}$ In contrast to our study, Mahapatra et $a l^{6}$ showed that invasively assessed $\mathrm{C}_{\mathrm{PA}}$ is a strong predictor of survival in patients with idiopathic $\mathrm{PAH}$ (iPAH). In our patients, mean $\mathrm{C}_{\mathrm{PA}}$ was lower $(1.0(0.8 ; 1.4) \mathrm{mL} /$ $\mathrm{mm} \mathrm{Hg})$ than in the iPAH cases of Mahapatra et al $(1.43 \pm 0.73$ (range $0.40-3.77) \mathrm{mL} / \mathrm{mm} \mathrm{Hg}$ ). ${ }^{9}$

Based on our data, one could hypothesise that an important component of $\mathrm{C}_{\mathrm{PA}}$ is located in surgically accessible pulmonary arteries in a majority of patients represented in the upper left quadrant of figure 4 . The relationship between PVR and $\mathrm{C}_{\mathrm{PA}}$ is hyperbolic, with a proportionally more important decrease of $\mathrm{C}_{\mathrm{PA}}$ at close to normal PVR, and little further decrease of $\mathrm{C}_{\mathrm{PA}}$ in severe $\mathrm{PH}$. The RC-time, which describes the exponential decay of the PAP during diastole, was 0.72 at baseline, slightly higher than previously reported in a smaller population of CTEPH patients $(0.49 \mathrm{~s}) .{ }^{16}$ The product of PVR and $\mathrm{C}_{\mathrm{PA}}$ did not change (figure $3 \mathrm{~A}-\mathrm{C}$ ) which is in concordance with findings of patients under PAH-specific treatments. ${ }^{8} 9$ Searching for cases in whom proximal compliance improved more than resistance, we chose to represent individual cases as vectors from the origin. The majority of vectors point to the top-left quadrant confirming that if PVR decreases, $\mathrm{C}_{\mathrm{PA}}$ increases concordantly, indicating a significant contribution of major vessel disobliteration to the improvement of vascular compliance in CTEPH. Those patients in whom PVR and $\mathrm{C}_{\mathrm{PA}}$ are dissociated appear to experience worse outcomes $(100 \%$ persistent/recurrent $\mathrm{PH}$ in the right upper quadrant of figure 4 , and $67 \%$ persistent/recurrent $\mathrm{PH}$ in the left lower quadrant of figure 4). Causes may be a stiff and obstructed distal microcirculatory compartment or residual thrombus.

In the outcome study we analysed the effects of haemodynamic changes on long-term survival/freedom of lung transplantation. CTEPH is a dual vascular disorder, with a major vessel disease component that can be addressed by surgical PEA as well as a microvascular disease (secondary vasculopathy). Secondary vasculopathy of CTEPH is indistinguishable from 
pulmonary vascular lesions seen in $\mathrm{PAH}^{19}$ and affects pulmonary arteries measuring less than $200 \mu \mathrm{m}$ in diameter, contributing to higher PVR and increased postoperative mortality. It has been difficult to compartmentalise resistance prior to PEA, that is, to predict which proportion of PVR is due to major vessel obstruction and which is due to small vessel disease. ${ }^{20} 21 \mathrm{~A}$ preoperative PVR above 1000 dynes.s. $\mathrm{cm}^{-5}$ has generally been labelled as an important clinical risk factor, underlying a mortality rate of $10.1 \%,{ }^{1}$ and is possibly a marker for significant small vessel disease. In the international prospective CTEPH registry, ${ }^{2}$ patients presenting with baseline PVR between 800 and 1000 dynes.s. $\mathrm{cm}^{-5}$ had mortality rates (inhospital and 1-year) of $14.4 \%$ (15 of 104 patients), while patients with PVR $>1200$ dynes.s. $\mathrm{cm}^{-5}$ had mortality rates of $23.4 \%$. While a baseline PVR $>1000$ dynes.s.cm ${ }^{-5}$ was a weak predictor of survival, preoperative PVR as a continuous variable did not appear as a multivariate predictor of 1-year-survival, similar to what was seen in the CTEPH registry. ${ }^{2}$ Furthermore, more recent studies have documented a significant reduction in post-PEA mortality even in patients with PVR $>1000$ dynes.s. $\mathrm{cm}^{-5}$ at baseline. $^{4}$ By contrast, PVR measured immediately postoperative $^{11}$ had a significant influence on long-term outcome $(\mathrm{p}<0.0001)$, at a threshold of 590 dynes.s.cm ${ }^{-5}$. Similar cut-offs $\left(500\right.$ dynes.s. $\left.\mathrm{cm}^{-5}\right)$ were predictive of 30 -day outcomes $(\mathrm{p}<0.001)$ in the study by Jamieson and coworkers. ${ }^{1}$ Our data demonstrate that patients with immediate postoperative PVR $<290$ dynes.s. $\mathrm{cm}^{-5}$ had excellent outcomes beyond 10 years FU.

\section{Limitations}

The relatively small study size and the single-centre design count as limitations. In addition, PEA specimens were not available from all patients and assessments were semiquantitative. Outcomes were influenced by a centre-specific 'learning curve' that is significant in this technically difficult surgical procedure. Furthermore, we did not systematically assess residual thrombus by postoperative imaging.

\section{CONCLUSIONS}

The data support the value of a complete and bilateral surgical endarterectomy, and the need for a final haemodynamic assessment in the intensive care unit prior to removal of the Swan-Ganz catheter to assess postoperative PVR. PVR is the key haemodynamic parameter for predicting prognosis in CTEPH patients undergoing PEA. The outcome of operable CTEPH is best predicted immediately after PEA, based on PVR and the characteristics of the surgical specimen.

Contributors NS-S and IML designed the protocol, submitted ethics application, included patients and treated patients. RS-K collected the database. ST, PN and WK performed pulmonary endarterectomy. GM and CG performed the analysis of PEA specimens. GH and CG prepared statistical analyses and the graphs. NS-S and IML drafted the work.

Funding Funded by an educational grant from Bayer (trial number 15662).

Competing interests Irene Marthe Lang and Nika Skoro-Sajer have relationships with drug companies including AOPOrphan Pharmaceuticals, Actelion, Bayer, Astra-Zeneca, Servier, Cordis, Medtronic, GSK, Novartis, Pfizer and United Therapeutics. In addition, Irene Marthe Lang is an investigator in trials involving these companies, with relationships including consultancy service, research grants and membership of scientific advisory boards.
Patient consent Obtained.

Ethics approval IRB Committee Name: Ethik Kommission der Medizinischen Universität Wien.

Provenance and peer review Not commissioned; externally peer reviewed.

Open Access This is an Open Access article distributed in accordance with the Creative Commons Attribution Non Commercial (CC BY-NC 3.0) license, which permits others to distribute, remix, adapt, build upon this work non-commercially, and license their derivative works on different terms, provided the original work is properly cited and the use is non-commercial. See: http://creativecommons.org/ licenses/by-nc/3.0/

\section{REFERENCES}

1 Jamieson SW, Kapelanski DP, Sakakibara N, et al. Pulmonary endarterectomy: experience and lessons learned in 1,500 cases. Ann Thorac Surg 2003;76:1457-62; discussion 1462-1454.

2 Mayer $E$, Jenkins D, Lindner J, et al. Surgical management and outcome of patients with chronic thromboembolic pulmonary hypertension: results from an international prospective registry. J Thorac Cardiovasc Surg 2011;141:702-10.

3 Thistlethwaite PA, Kaneko K, Madani MM, et al. Technique and outcomes of pulmonary endarterectomy surgery. Ann Thorac Cardiovasc Surg 2008;14:274-82.

4 Madani MM, Auger WR, Pretorius V, et al. Pulmonary endarterectomy: recent changes in a single institution's experience of more than 2,700 patients. Ann Thorac Surg 2012;94:97-103; discussion 103.

5 Stevens GR, Garcia-Alvarez A, Sahni S, et al. RV dysfunction in pulmonary hypertension is independently related to pulmonary artery stiffness. JACC CardiovasC Imaging 2012;5:378-87.

6 Mahapatra S, Nishimura RA, Sorajja P, et al. Relationship of pulmonary arterial capacitance and mortality in idiopathic pulmonary arterial hypertension. J Am Coll Cardiol 2006;47:799-803

7 Bonderman D, Martischnig AM, Vonbank K, et al. Right ventricular load at exercise is a cause of persistent exercise limitation in patients with normal resting pulmonary vascular resistance after pulmonary endarterectomy. Chest 2011;139:122-7.

8 Lankhaar JW, Westerhof N, Faes TJ, et al. Pulmonary vascular resistance and compliance stay inversely related during treatment of pulmonary hypertension. Eur Heart J 2008;29:1688-95.

9 Lankhaar JW, Westerhof N, Faes TJ, et al. Quantification of right ventricular afterload in patients with and without pulmonary hypertension. Am J Physiol Heart Circ Physiol 2006;291:H1731-1737.

10 Klepetko W, Mayer E, Sandoval J, et al. Interventional and surgical modalities of treatment for pulmonary arterial hypertension. J Am Coll Cardiol 2004;43:73S-80S.

11 Skoro-Sajer N, Hack N, Sadushi-Kolici R, et al. Pulmonary vascular reactivity and prognosis in patients with chronic thromboembolic pulmonary hypertension: a pilot study. Circulation 2009;119:298-305.

12 Robin X, Turck N, Hainard A, et al. pROC: an open-source package for R and S+ to analyze and compare ROC curves. BMC Bioinform 2011;12:77.

13 Team RDC. R: A language and environment for statistical computing. R Foundation for Statistical Computing, Vienna, Austria. 2011; ISBN: 3-900051-07-0. http://www. R-project.org

$14 \mathrm{Kim} \mathrm{NH}$. Assessment of operability in chronic thromboembolic pulmonary hypertension. Proc Am Thorac Soc 2006;3:584-8.

15 Thistlethwaite PA, Mo M, Madani MM, et al. Operative classification of thromboembolic disease determines outcome after pulmonary endarterectomy. J Thorac Cardiovasc Surg 2002;124:1203-11.

16 Saouti N, Westerhof N, Helderman F, et al. RC time constant of single lung equals that of both lungs together: a study in chronic thromboembolic pulmonary hypertension. Am J Physiol Heart Circ Physiol 2009;297:H2154-60.

17 Westerhof N, Bosman F, De Vries CJ, et al. Analog studies of the human systemic arterial tree. J Biomech 1969;2:121-43.

18 Stergiopulos N, Segers P, Westerhof N. Use of pulse pressure method for estimating total arterial compliance in vivo. Am J Physiol 1999;276:H424-8.

19 Moser KM, Bloor CM. Pulmonary vascular lesions occurring in patients with chronic major vessel thromboembolic pulmonary hypertension. Chest 1993;103:685-92.

20 Toshner $\mathrm{M}$, Suntharalingam J, Fesler $\mathrm{P}$, et al. Occlusion pressure analysis role in partitioning of pulmonary vascular resistance in CTEPH. Eur Respir $J$ 2012;40:612-17.

21 Kim NH, Fesler P, Channick RN, et al. Preoperative partitioning of pulmonary vascular resistance correlates with early outcome after thromboendarterectomy for chronic thromboembolic pulmonary hypertension. Circulation 2004;109:18-22. 